\title{
Existence and Stability Estimate for the Solution of the Ageing Hereditary Linear Viscoelasticity Problem
}

\author{
Julia Orlik \\ Fraunhofer-Institut für Techno- und Wirtschaftsmathematik, \\ Gottlieb-Daimler Straße 49 D, 67663 Kaiserslautern, Germany \\ Correspondence should be addressed to Julia Orlik, orlik@itwm.fhg.de
}

Received 19 January 2009; Accepted 23 April 2009

Recommended by Victor Zvyagin

The paper is concerned with the existence and stability of weak (variational) solutions for the problem of the quasistatic evolution of a viscoelastic material under mixed inhomogenous Dirichlet-Neumann boundary conditions. The main novelty of the paper relies in dealing with continuous-in-time weak solutions and allowing nonconvolution and weak-singular Volterra's relaxation kernels.

Copyright (C) 2009 Julia Orlik. This is an open access article distributed under the Creative Commons Attribution License, which permits unrestricted use, distribution, and reproduction in any medium, provided the original work is properly cited.

\section{Introduction}

Our work deals with mathematical problems arising from considering the behavior of hereditary viscoelastic solids. These result in the system of elliptic partial differential equations in space variables, whose coefficients are Volterra integral operators of the second kind in time, which allow for weak-singular kernels. In Sections 2 and 3 a general mathematical model of the boundary value problem of the inhomogeneous hereditary ageing viscoelasticity is given in the classical and weak formulations. The main result of this section, Theorem 3.2, proves the existence and uniqueness of the solution to the general problem of the hereditary ageing viscoelasticity with mixed boundary conditions. The proof of the main results is shifted to the end of the paper, since it is based on the main result of Section 4 applied to the class of continuous Banach-valued functions with values in the Sobolev spaces. Section 4 can be considered separately of the mechanical background of the problem and can be interesting from the point of view of "Linear Volterra integral operators in Banach spaces". It includes definition of classes of Volterra operators with operator-valued kernels acting in the space of continuous Banach-valued functions. We also present some well-known 
results from the theory of Bochner's integral and their extension to the introduced classes. Lemma 5.4 delivers statements about the spectral radius and representation as a series for scalar Volterra operators. Theorem 6.2 delivers the main result of this section. It gives a statement about the solvability of Volterra's integral equation in the space of continuous functions with values in Banach spaces. The idea of its proof is the reduction of a statement for Banach-valued functions to the corresponding statement for real-valued functions. Similar ideas were proposed in [1] for Volterra operators with kernels depending on a parameter. The main principle for obtaining this purpose is given by Theorem 4.1. The question of the solvability of nonconvolutional Volterra integral equations in Banach spaces was considered in [2-10], but under somewhat stricter assumptions for the kernel classes and almost all the proofs were given by using semigroup theory or fixed-point arguments. That is, in [2] the same problem is considered but the solvability proof restricts on a special kind of the kernel's weak singularity and is based on the fixed-point argument. The work in [6] allows for weak singular kernels, but again of the same as in [2] special type, $(t-\tau)^{-\alpha}$. This allows to get a simple estimate for the partial sum of the Neumann series in the form of an explicit formula, which shows its convergence. In the work [8], the kernels are supposed to be continuous on the whole time interval. References $[3,5]$ consider evolutional integrodifferential equations with weak singular kernels, but such equations can be transformed by partial integration to the Volterra integral equation with bounded kernels. While we are looking for the solution in the space of continuous Banach-valued functions, [7] proceeds in $L^{p}$-spaces, $p<\infty$. Papers $[4,9,10]$ first assume the existence of the resolvent of the operator kernel part, that is, that its spectral radius is zero, and second do not permit the dependence of the operator kernel part on both time-variables separately.

\section{Definition of the Problem}

We consider a linear viscoelastic and aging (of the non-convolutional integral type) body, which is subjected to some external loading. We denote the domain occupied by the body by $\Omega \subset \mathbb{R}^{n}$, which is a Lipschitz domain.

We are going to consider the equilibrium equations for such a solid. We would like to recall that viscoelastic solid is still a solid, its deformation is slow and we restrict ourselves to the quasi-static, that is, classical for the solid mechanics statement of problem, without the inertial term. A summation from 1 to $n$ over repeating indices is assumed in all the present work, unless opposite is stated:

$$
\frac{\partial}{\partial x_{h}}\left(\left(a_{i j_{0}}^{h k}(x, t)+a_{i j}^{h k}(x) \star\right) \frac{\partial u_{j}(x, t)}{\partial x_{k}}\right)=-f_{i_{0}}(x, t), \quad x \in \Omega
$$

$i, j, h, k=1,2, \ldots, n$, with boundary conditions:

$$
\begin{gathered}
u_{i}(x, t)=\psi_{i}(x, t), \quad x \in \partial \Omega_{u \prime} \\
\left(\left(a_{i j 0}^{h k}(x, t)+a_{i j}^{h k}(x) \star\right) \frac{\partial u_{j}(x, t)}{\partial x_{k}}\right) n_{h}(x)=\phi_{i}(x, t), \quad x \in \partial \Omega_{\sigma},
\end{gathered}
$$


holding for any $t \in[0, T]$. Here

$$
\left(a_{i j}^{h k}(x) \star e_{k}^{j}\right)(t):=\int_{0}^{t} a_{i j}^{h k}(x, t, \tau) \cdot e_{k}^{j}(x, \tau) d \tau
$$

are Volterra integral operators with kernels $a_{i j}^{h k}(x, t, \tau) ; a_{i j}^{h k}(x, t)$ are instantaneous elastic coefficients (out-of-integral terms) and $\underline{a}_{i j}^{h k}(x):=a_{i j}^{h k}(x, t)+a_{i j}^{h k}(x) \star ; f_{i_{0}}$ are components of a vector of external forces; $\phi_{i}(x, t)$ are components of a vector of boundary traction on the part $\partial \Omega_{\sigma}$ of the external boundary; $\psi(x, t)$ are components of the displacement vector on the rest part $\partial \Omega_{u}$ of the boundary. All functions are supposed to be continuous w.r.t. $t \in[0, T]$ and sufficiently smooth w.r.t. $x$ in domain $\Omega$ (for performing a partial integration). The whole viscoelastic operator tensor $\left(\underline{a}_{i j}^{h k}(x)\right)_{n \times n}^{n \times n}$ is assumed to be symmetric at each point $x \in \Omega$ :

$$
\underline{a}_{i j}^{h k}(x)=\underline{a}_{j i}^{k h}(x)=\underline{a}_{h j}^{i k}(x)=\underline{a}_{i k}^{h j}(x)
$$

The tensor $\left(a_{i j_{0}}^{h k}(x, t)\right)_{n \times n}^{n \times n}$ is additionally positive definite, with elements bounded at each point $x \in \Omega$

$$
c_{0} \eta_{k}^{j} \eta_{k}^{j} \leq a_{i j_{0}}^{h k}(x, t) \eta_{h}^{i} \eta_{k}^{j} \leq C_{0} \eta_{k}^{j} \eta_{k^{\prime}}^{j}
$$

for all $\eta_{k}^{j}=\eta_{j}^{k} \in \mathbb{R}$ and $t \in[0, T]$ where the constants $0<c_{0} \leq C_{0}<\infty$ are independent of $x$ and $t$. For isotropic materials $\underline{a}_{i j}^{h k}=\underline{\lambda} \delta_{h i} \delta_{k j}+\underline{\mu} \delta_{i j} \delta_{h k}+\underline{\mu} \delta_{i k} \delta_{h j}$.

Example 2.1. (i) Often, the kernels $a_{i j}^{h k}(x, t, \tau)$ are of the convolution type and are taken in the exponential form

$$
a_{i j}^{h k}(x, t, \tau)= \begin{cases}\sum_{p=1}^{m} \alpha_{i j}^{h k}(x) e^{-\beta_{p}(x)(t-\tau)}, & \text { if } t \geq \tau, \\ 0, & \text { if } t<\tau,\end{cases}
$$

where the $\beta_{p}$ are piece-wise continuous functions, often just constants, and the $\alpha_{i j_{p}}^{h k}(x)$ are piece-wise continuous functions for $x \in \Omega$.

(ii) The $a_{i j}^{h k}(x, t, \tau)$ may also be kernels of the Abel type (typical example for the relaxation kernels of concrete and cement):

$$
a_{i j}^{h k}(x, t, \tau)= \begin{cases}A_{i j_{1}}^{h k}(x, t, \tau)(t-\tau)^{-\alpha}+A_{i j_{2}}^{h k}(x, t, \tau)(\tau)^{-\beta} & \\ +A_{i j_{3}}^{h k}(x, t, \tau) t^{-\gamma}, & \text { if } t \geq \tau, \\ 0, & \text { otherwise }\end{cases}
$$

with $0 \leq \alpha, \beta, \gamma<1$. The $A_{i j_{p}}^{h k} p=1,2,3$, are continuous in $t$ and $\tau$, and piece-wise continuous in $x \in \Omega$. 


\section{Weak Problem Formulation and Main Results}

In order to obtain the variational formulation, we multiply (2.1) by test functions $v_{i}(x) \in$ $H_{0}^{1}\left(\Omega, \partial \Omega_{u}\right), i=1, \ldots, n$, where $H_{0}^{1}\left(\Omega, \partial \Omega_{u}\right):=\left\{v \in H^{1}(\Omega): v(x)=0, x \in \partial \Omega_{u}\right\}$, and integrate over the whole domain $\Omega$. Integrating by parts and taking into account boundary condition (2.2), we obtain the following variational problem.

Find $u_{j} \in H^{1}(\Omega), j=1, \ldots, n$, satisfying (2.2) and

$$
\begin{array}{r}
\int_{\Omega} \underline{a}_{i j}^{h k} \frac{\partial u_{j}}{\partial x_{k}} \frac{\partial v_{i}}{\partial x_{h}} d x=l(v), \quad l(v):=\int_{\Omega} f_{i 0} v_{i} d x+\int_{\partial \Omega_{\sigma}} \phi_{i} v_{i} d s, \\
\forall v_{i} \in H_{0}^{1}\left(\Omega, \partial \Omega_{u}\right), i=1, \ldots, n .
\end{array}
$$

Definition 3.1 (General weak formulation). Consider the matrix of instantaneous elastic coefficients $\left(a_{i j}^{h k}\right)_{n \times n} \in C\left([0, T] ; \mathbf{L}^{\infty}(\Omega)\right)$, the relaxation operators $\left(a_{i j}^{h k} \star\right)_{n \times n}$, such that $a_{i j}^{h k}(t, \tau)=0 \forall \tau>t$, and $a_{i j}^{h k} \in C\left([0, T] ; L^{\infty}(\Omega)\right)$, and $f_{0}:=\left(f_{i 0}\right)_{n} \in C\left([0, T] ; \mathbf{H}^{-1}(\Omega)\right)$, the whole viscoelastic operator $\underline{a}_{i j}^{h k}=a_{i j 0}^{h k}+a_{i j}^{h k} \star$, the boundary tractions $\phi:=\left(\phi_{i}\right)_{n} \in$ $C\left([0, T] ; \mathbf{H}^{-1 / 2}\left(\partial \Omega_{\sigma}\right)\right)$, and boundary displacements $\psi:=\left(\psi_{i}\right)_{n} \in C\left([0, T] ; \mathbf{H}^{1 / 2}\left(\partial \Omega_{u}\right)\right)$.

One defines a weak solution of problem (2.1)-(2.3) as a vector-valued function $u \in$ $C\left([0, T] ; \mathbf{H}^{1}(\Omega)\right)$, which can be represented in the form $u=\widehat{u}+\widetilde{\psi}$, where $\widetilde{\psi} \in C\left([0, T] ; \mathbf{H}^{1}(\Omega)\right)$ satisfies $\left(\left.\tilde{\psi}\right|_{\partial \Omega_{u}}\right)=\psi$ and $\widehat{u}_{i} \in C\left([0, T] ; H_{0}^{1}\left(\Omega, \partial \Omega_{u}\right)\right), i=1, \ldots, n$, satisfies the integral identity

$$
[\underline{a}(\widehat{u}, v)](t):=\int_{\Omega}\left[\underline{a}_{i j}^{h k} \frac{\partial \widehat{u}_{j}}{\partial x_{k}}\right](t) \frac{\partial v_{i}}{\partial x_{h}} d x=\widehat{l}(v)(t) \quad \forall t \in[0, T]
$$

for any $v_{i} \in H_{0}^{1}\left(\Omega, \partial \Omega_{u}\right)$. The right hand-side of (3.2) is, for all $t \in[0, T]$, a linear functional on the $\mathbf{H}_{0}^{1}\left(\Omega, \partial \Omega_{u}\right)$

$$
\widehat{l}(v)(t):=\int_{\Omega}\left(f_{i 0}(t) v_{i}-\left[\underline{a}_{i j}^{h k} \frac{\partial \tilde{\psi}_{j}}{\partial x_{k}}\right](t) \frac{\partial v_{i}}{\partial x_{h}}\right) d x+\int_{\partial \Omega_{\sigma}} \phi_{i}(t) v_{i} d s
$$

The space of linear bounded functionals on $\mathbf{H}_{0}^{\mathbf{1}}\left(\Omega, \partial \Omega_{u}\right)$ is denoted by $H^{-1}(\Omega)$.

We denote further $a_{0}(\widehat{u}, v)(t):=\int_{\Omega} a_{i j}^{h k}(x, t)\left(\partial \widehat{u}_{j}(x, t) / \partial x_{k}\right)\left(\partial v_{i}(x) / \partial x_{h} d x\right), a(\widehat{u}, v)(t$, $\tau):=\int_{\Omega} a_{i j}^{h k}(x, t, \tau)\left(\partial \hat{u}_{j}(x, \tau) / \partial x_{k}\right)\left(\partial v_{i}(x) / \partial x_{h}\right) d$. Obviously, $[\underline{a}(\widehat{u}, v)](t)=a_{0}(\widehat{u}, v)(t)+$ $\int_{0}^{t} a(\widehat{u}, v)(t, \tau) d \tau$.

Note that $a_{0}(\widehat{u}, v)$ and $a(\widehat{u}, v)$ are bilinear forms on $\mathbf{H}_{0}^{1}\left(\Omega, \partial \Omega_{u}\right)$ for every $t$ and almost every $\tau$. We can rewrite the weak formulation (3.2) as follows:

$$
a_{0}(\widehat{u}, v)(t)+\int_{0}^{t} a(\widehat{u}, v)(t, \tau) d \tau=l(v)(t), \quad t \in[0, T]
$$


Let us finally rewrite (3.4) in the operator form. For this purpose we introduce the following notations:

$$
A_{0 x} \widehat{u}:=a_{0}(\widehat{u}, \cdot), \quad A_{x} \widehat{u}:=a(\widehat{u}, \cdot), \quad L(t):=l(v)(t) .
$$

$A_{0 x}(t), A_{x}(t, \tau): \mathbf{H}_{0}^{1}\left(\Omega, \partial \Omega_{u}\right) \rightarrow H^{-1}(\Omega)$ for all fixed $t$ and almost all $\tau \in[0, T]$. Now we can represent (3.2) in the form $\underline{A} \widehat{u}=L(t)$, where $\underline{A}$. is an infinite dimensional integro-differential linear operator $\underline{A}=A_{0 x} \cdot+A_{x} \star$ and the weak problem formulation (3.2) takes the form

$$
A_{0 x}(t) \widehat{u}(t)+\left[A_{x} \star \widehat{u}\right](t)=L(t) .
$$

Equation (3.6) provides the most general form of the time-space integro-differential dependencies of the considered problem. The following theorem is used as an auxiliary result for showing the solvability of such equation.

Theorem 3.2 (Data stability). Let $\Omega \subset \mathbb{R}^{n}$ be a Lipschitz domain and $\partial \Omega_{u} \subseteq \partial \Omega$, let $A_{0 x} \in C\left([0, T] ; \mathcal{L}\left(\mathbf{H}_{0}^{1}\left(\Omega, \partial \Omega_{u}\right), H^{-1}\right)\right)$, and let $A_{0 x}(t)$ be boundedly-invertible uniformly in $[0, T]$, $A_{x}(t, \tau)=0 \forall \tau>t, A_{x} \in C\left([0, T] ; L^{1}\left([0, T], \mathcal{L}\left(\mathbf{H}_{0}^{1}\left(\Omega, \partial \Omega_{u}\right), H^{-1}\right)\right)\right)$, and $f \in C\left([0, T] ; H^{-1}\right)$. Then there exists a unique global solution $u$ of the problem

$$
A_{0 x}(t) u(t)+\left[A_{x} \star u\right](t)=f(t)
$$

in $C\left([0, T] ; \mathbf{H}_{0}^{1}\left(\Omega, \partial \Omega_{u}\right)\right)$, which depends continuously on $f$, that is,

$$
\|u\|_{C\left([0, T] ; H^{1}(\Omega)\right)} \leq C_{1}\|f\|_{C\left([0, T] ; H^{-1}\right)},
$$

where the constant $C_{1}$ is independent of $f$, and if $\left\|A_{0 x}^{-1}(t)\right\|_{\mathcal{L}\left(H^{-1}, H^{1}\right)} \leq 1 / c_{0}$, then

$$
C_{1} \leq \tilde{C}\left(\frac{1}{C_{0}} \max _{i, j, h, k}\left\|a_{i j}^{h k}(t, \tau)\right\|_{C\left(\left([0, T], L^{1}\left([0, T], L^{\infty}(\Omega)\right)\right)\right)}\right),
$$

where $\tilde{C}$ is some real-valued function, independent of $f$.

The rest of the paper is aimed to prove this theorem. The final proof of this theorem as well as of two following lemmas can be found in the Appendix. Since we are searching for the weak solution in the form $u=\widehat{u}+\widehat{\psi}$, where both $\widehat{u}$ and $\widehat{\psi}$ are in $C\left([0, T] ; H^{1}(\Omega)\right)$, then we can claim that our solution exists and is unique.

Lemma 3.3. Let $\Omega$ be a Lipschitz domain in $\mathbb{R}^{n}$, the instantaneous elastic (out-of-integral) coefficients $a_{i j_{0}}^{h k} \in C\left([0, T] ; L^{\infty}(\Omega)\right.$ ) satisfy the positivity condition (the first of (2.6)) with a constant $c_{0}$, and 
the relaxation kernels $a_{i j}^{h k} \in C\left(\left([0, T], L^{1}\left([0, T], L^{\infty}(\Omega)\right)\right)\right)$. Then

(i) $A_{0 x}$ belongs to $C\left([0, T] ; \mathcal{L}\left(\mathbf{H}_{0}^{1}\left(\Omega, \partial \Omega_{u}\right), H^{-1}\right)\right)$, and $A_{0 x}(t)$ has an inverse operator $A_{0 x}^{-1}(t) \forall t \in[0, T]$. This inverse operator is uniformly bounded in $[0, T]$, that is, the following estimate:

$$
\left\|A_{0 x}^{-1}(t)\right\|_{\mathcal{L}\left(H^{-1}, H^{1}\right)} \leq \frac{1}{c_{0}}
$$

holds for any $t \in[0, T]$, and $c_{0}$ is independ on $t$;

(ii) $A_{x}(t, \tau)$ satisfies the following estimate:

$$
\left\|A_{x}(t, \tau)\right\|_{\mathcal{L}\left(H^{1}(\Omega), H^{-1}\right)} \leq \max _{i, j, h, k}\left\|a_{i j}^{h k}(t, \tau)\right\|_{L^{\infty}(\Omega)^{\prime}}
$$

for all t and a.a $\tau \in[0, T]$. Furthermore, the condition $a_{i j}^{h k} \in C\left(\left([0, T], L^{1}\left([0, T], L^{\infty}(\Omega)\right)\right)\right.$ implies that $A_{x} \in C\left(\left([0, T], L^{1}\left([0, T], \mathcal{L}\left(\mathbf{H}_{0}^{1}\left(\Omega, \partial \Omega_{u}\right), H^{-1}\right)\right)\right)\right.$.

Lemma 3.4. In both cases of Example 2.1, $a_{i j}^{h k} \in C\left(\left([0, T], L^{1}\left([0, T], L^{\infty}(\Omega)\right)\right)\right)$, that is, are the Volterra kernels.

\section{Volterra Integral Operators in Banach Spaces}

The following theorem (which can be found, e.g., in [11, section 7.2]) delivers the tool, which we will multiply use for obtaining the main results of this section.

Theorem 4.1. A strongly-measurable Banach-valued function $f(s)$ is Bochner-integrable, if its norm in the corresponding Banach-space is Lebesgue integrable, and it follows (see [12, page 133]), $\left\|\int_{J} f(s) \lambda(d s)\right\|_{X} \leq \int_{J}\|f(s)\|_{X} \lambda(d s)$. $L^{\infty}(J, X)$.

We introduce the notation $\mathcal{S}(J, X)$, which should be interpreted as either $C(J, X)$ or

Corollary 4.2. Let $J \subset \mathbb{R}$ be a finite segment $(J=[0, T]), X$ and $Z$ be Banach-spaces, and $K \in$ $L^{1}(J, \mathcal{L}(X, Z))$. Then $K(\cdot) g(\cdot) \in L^{1}(J, Z)$ for all $g \in \mathcal{S}(J, X)$.

Proof. Each $g \in C(J, X)$ is Bochner integrable, hence, strongly measurable, and each $g \in$ $L^{\infty}(J, X)$ is strongly measurable, according to definition of the space $L^{\infty}(J ; X)$. Thus we can find disjoint sets $B_{i, n} \subset J$ and finitely-valued functions $g_{n}(\tau):=\sum_{i=1}^{n} g_{i, n} \chi_{B_{i, n}}(\tau), \forall \tau \in J$, such that $\lim _{n \rightarrow \infty}\left\|g_{n}(\tau)-g(\tau)\right\|_{X}=0$ a.e. on $J$. Here $X_{B_{i, n}}(\tau)$ is the characteristic function of $B_{i, n}$. Thus

$$
\left\|K(\tau) g_{n}(\tau)-K(\tau) g(\tau)\right\|_{Z} \leq\|K(\tau)\|_{\mathcal{L}(X, Z)}\left\|g_{n}(\tau)-g(\tau)\right\|_{X} \longrightarrow 0 \quad \text { as } n \longrightarrow \infty
$$

for almost all $\tau \in J$. $K$ is strongly measurable on $J ; K(\tau) g_{n}(\tau):=\sum_{i=1}^{n} K(\tau) g_{i, n} \chi_{B_{i, n}}(\tau), \forall \tau \in J$, where $K(\tau) g_{i, n}$ is, for all $n$, a strongly measurable $Z$-valued function, owing to [12, Chapter 5 , Corollary 2], and $X_{B_{i, n}}: \quad J \rightarrow \mathbb{R}$. Owing to [13, the Appendix, Proposition (9a)], $K(\tau) g_{n}(\tau)$ 
is strongly measurable on $J$. Then, according to the convergence theorem of Egorov, in a view of (4.1) (see [13, page 1013]), $K(\tau) g(\tau)$ is also strongly measurable on $J$.

Owing to the fact that $\|K(\cdot) \mathcal{L}\|_{(\mathrm{X}, \mathrm{Z})} \in L^{1}(J)$, we have,

$$
\begin{aligned}
\int_{J}\|K(\tau) g(\tau)\|_{Z} d \tau & \leq \int_{J}\|K(\tau)\|_{\mathcal{L}(X, Z)}\|g(\tau)\|_{X} d \tau \\
& \leq\|g\|_{\mathcal{S}(J, X)} \int_{J}\|K(\tau)\|_{\mathcal{L}(X, Z)} d \tau<\infty .
\end{aligned}
$$

Using Theorem 4.1 completes the proof.

Since for $J=[0, T], C(J, X) \hookrightarrow L^{\infty}(J, X)$, it would be enough to consider only $L^{\infty}$ instead of $\mathcal{S}$ in Corollary 4.2 and also in further considerations. Nevertheless, we often will need this theory specifically for continuous Banach-valued functions in the main part of this work. Therefore it is reasonable to keep $\mathcal{S}$.

Now we define a class of Banach-valued Volterra operators.

Definition 4.3. Let $X, Z$ be Banach spaces and $J \subset \mathbb{R}$ be a finite segment $[0, T], 0 \leq T<\infty$, or

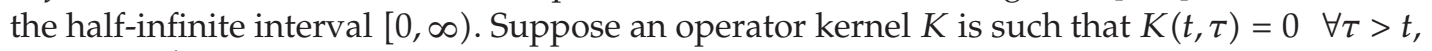
$K \in C\left(J, L^{1}(J, \mathcal{L}(X, Z))\right)$, that is,

(i) $K:(t, \tau) \rightarrow \mathcal{L}(X, Z), \forall t \in J$ and almost all $\tau \in J, K(t, \tau)$ is strongly measurable w.r.t. $\tau$, for all $t \in J$, and $\|K(t, \tau)\|_{\mathcal{L}(X, Z)}$ is integrable w.r.t. $\tau$ for all $t$ in $J$, that is, $\int_{J}\|K(t, \tau)\|_{\mathcal{L}(\mathrm{X}, \mathrm{Z})} d \tau<\infty \forall t \in J ;$

(ii) $\int_{J}\|K(t+\Delta t, \tau)-K(t, \tau)\|_{\mathcal{L}(\mathrm{X}, \mathrm{Z})} d \tau \rightarrow$ as $\Delta t \rightarrow 0 \forall t, t+\Delta t \in J$.

Call $K \star$ defined by $g \mapsto(K \star g)(t):=\int_{J} K(t, \tau) g(\tau) d \tau$, for $g \in \mathcal{S}(J, X)$ a Volterra integral operator. The set of all Volterra integral operators is denoted by $V(C ; J ; \mathcal{L}(X, Z))$.

Remark 4.4. Observe that, as soon as $J$ is closed and bounded (i.e., compact), condition (ii) of Definition 4.3 implies that the following holds:

$$
|\|K\||_{V(C ; J ; \mathcal{L}(X, Z))}:=\sup _{t \in J} \int_{J}\|K(t, \tau)\|_{\mathcal{L}(X, Z)} d \tau<\infty .
$$

We will call in such a case $\|K\| \|_{V(C ; J ; \mathcal{L}(X, Z))}$ the kernel norm of the operator $K \star$.

Corollary 4.5. Let $J=[0, T]$, and let $X, Z$ be Banach spaces. If $K \star \in V(C ; J ; \mathcal{L}(X, Z)), g \in$ $\mathcal{S}(J, X)$, one can view $f(t, \tau):=K(t, \tau) g(\tau)$ also as an element of $C\left(J, L^{1}(J, Z)\right)$.

Proof. The fact that $f(t, \cdot) \in L^{1}(J, Z)$ follows from Corollary 4.2. Let us show the continuity: $\|K(t+\Delta t, \cdot) g(\cdot)-K(t, \cdot) g(\cdot)\|_{L^{1}(J, Z)}=\int_{J}\|K(t+\Delta t, \tau) g(\tau)-K(t, \tau) g(\tau)\|_{Z} d \tau \leq$ $\int_{J}\|(K(t+\Delta t, \tau)-K(t, \tau))\|_{\mathcal{L}(X, Z)}\|g(\tau)\|_{X} d \tau \leq\|g\|_{\mathcal{S}(J, X)} \int_{J}\|K(t+\Delta t)-K(t)\|_{\mathcal{L}(X, Z)} d \tau \rightarrow$ as $\Delta t \rightarrow 0 \forall t, t+\Delta t \in J$. For abbreviation, we will set $V(C ; J ; X):=V\left(C ; J ; \mathcal{L}\left(\mathbb{R}^{n}, X\right)\right)$. 
Corollary 4.6. Let $J \subset \mathbb{R}$ be a segment, $X$ and $Z$ be Banach-spaces, $K \star \in V(C ; J ; \mathcal{L}(X, Z))$. Then $\|K\|_{\mathcal{L}(\mathrm{X}, \mathrm{Z})^{\star}} \in V(C ; J ; \mathbb{R})$ and the following estimate

$$
\|K \star g\|_{Z} \leq\|K\|_{\mathcal{L}(X, Z)} \star\|g\|_{X}
$$

holds for all $g \in \mathcal{S}(J, X)$ and $t \in J$.

Proof. An operator $\left(\|K\|_{\mathcal{L}(X, Z)} \star \mathcal{u}\right)(t) \equiv \int_{J}\|K\|_{\mathcal{L}(X, Z)}(t, \tau) u(\tau) d \tau, \forall u \in \mathcal{S}(J)$ has the scalar kernel $\|K(t, \tau)\|_{\mathcal{L}(X, Z)}: J^{2} \rightarrow \mathbb{R}$, which obviously satisfies all requirements of Definition 4.3 , since $K \star \in V(C ; J ; \mathcal{L}(X, Z))$. The estimate (4.4) follows directly from Theorem 4.1.

Corollary 4.7 (from Theorem 4.1 in application to Definition 4.3). If $J$ is a finite segment and $X, Z$ are Banach spaces, then $V(C ; J ; \mathcal{L}(X, Z)) \hookrightarrow \mathcal{L}(\mathcal{S}(J, X), C(J, Z))$, that is any operator $K \star \in$ $V(C ; J ; \mathcal{L}(X, Z))$ can be identified with a bounded linear mapping from $\mathcal{S}(J, X)$ to $C(J, Z)$.

Proof. The continuity is implied from condition (ii) from Definition 4.3, the compactness of the interval and the boundedness of $\|g(\tau)\|_{X}$ by applying Theorem 4.1 (see proof to Corollary 4.5).

The boundedness in the $C(J, Z)$-norm follows from the continuity and the fact that $J$ is compact. And Theorem 4.1 delivers the continuity of the embedding

$$
\begin{aligned}
& \|K(t, s) \star\|_{\mathcal{L}(S(J, X), C(J, Z))}=\sup _{\|g\|_{S(J, X)} \leq 1} \int_{J}\|K(t, s) g(s) d s\|_{C(J, Z)} \\
& \leq \sup _{\|g\|_{S(J, X)} \leq 1} \sup _{t \in[0, T]} \int_{J}\|K(t, s) g(s)\|_{Z} d s \\
& \leq \sup _{\|g\|_{\mathcal{S}(, X)} \leq 1} \sup _{t \in[0, T]} \int_{J}\|K(t, s)\|_{\mathcal{L}(X, Z)}\|g(s)\|_{X} d s \\
& \leq \sup _{\|g\|_{\mathcal{S}(, X)} \leq 1}\left(\|K\|\left\|_{V(C ; J ; \mathcal{L}(X, Z))}\right\| g \|_{\mathcal{S}(J, X)}\right) \\
& \leq\|K\|_{V(C ; J ; \mathcal{L}(X, Z))} \text {. }
\end{aligned}
$$

\section{Some Auxiliary Results from the Spectral Theory}

In this section we give definitions of the spectrum and of the resolvent for an element of a Banach algebra with a unit used in [14, A.2.1 ].

Definition 5.1. The spectrum $\sigma(Q)$ of $Q$ from a Banach-algebra with a unit $I$ is the set of all $\lambda \in \mathbb{C}$ for which $(\lambda I-Q)^{-1}$ does not exist or is not bounded. Furthermore, for $\lambda \in \mathbb{C} \backslash \sigma(Q)$, $(\lambda I-Q)$ is invertible and the inverse $\underline{\widetilde{R}}(\lambda) \equiv(\lambda I-Q)^{-1}$ is called the resolvent of $Q$, and belongs to the same algebra. 
The following definition is known from in [14, A.2.1 ] or in [15, 18.8 ].

Definition 5.2. Let $\mathcal{A}$ be a Banach-algebra with unit, $Q \in \mathcal{A}$ and $\sigma(Q)$ be the spectrum of $Q$. Then one calls the number $\rho(Q)=\sup \{|\lambda| \mid \lambda \in \sigma(Q)\}$ the spectral radius of $Q$.

In [16, Theorem 3.6] about Neumann's series the following is proven.

Theorem 5.3. Let $T$ be a bounded linear operator over a Banach-space $X$, with

$$
\lim _{n \rightarrow \infty} \sup \left\|T^{n}\right\|_{\mathcal{L}(X)}^{1 / n}<1
$$

then $(I-T)^{-1} \in \mathcal{L}(X)$ can be represented as a series:

$$
(I-T)^{-1}=\sum_{j=0}^{\infty} T^{j}
$$

Now, we formulate and prove a lemma about the spectral radius and representation of a resolvent for scalar Volterra operators as Neuman's series. It prepares the basis for the main result of this section.

Lemma 5.4. Let $J \subset \mathbb{R}$ be a compact interval $(J=[0, T])$, and $Q \star \in V(C ; J ; \mathbb{C})$. Then there exists a resolvent $\underline{\tilde{R}}(\lambda)$ of $Q \star$ in $\mathcal{L}(\mathcal{S}([0, T], \mathbb{C}))$, given by the convergent series $\underline{\tilde{R}}(\lambda)=\sum_{j=0}^{\infty}\left((Q \star)^{j} / \lambda^{j+1}\right)$ for each $\lambda \in \mathbb{C} \backslash\{0\}$.

Proof. The class of kernels of Volterra operators from $V(C ; J ; \mathbb{C})$, on the compact interval forms a Banach algebra with product $\star$. This follows from [17, Theorem 9.5.3(ii)]. Such an algebra, owing to [18, Theorem 2, Section 20, Chapter 4], does not have a unit. According to $[19$, page 228], we can add an artificial unit to our algebra $\mathbb{A}$ kernels from $V(C ;[0, T] ; \mathbb{C})$. Owing to [17, Theorem 9.5.5(ii)], every kernel $Q, Q \star \in V(C ;[0, T] ; \mathbb{C})$ on some bounded interval $[0, T] \subset \mathbb{R}$ has an element $R$ (called there a resolvent of $Q$ ), which satisfies the following equality:

$$
R+Q \star R=R+R \star Q=Q
$$

such that $R \star \in V(C ;[0, T] ; \mathbb{C})$.

Obviously, if $Q \star \in V(C ;[0, T] ; \mathbb{C})$, then, for all $\lambda \in \mathbb{C} \backslash\{0\}, P \star \equiv-(1 / \lambda) Q \star$ belongs also to $V(C ;[0, T] ; \mathbb{C})$, and, hence, has a resolvent (in the sense of $[17])$ in $V(C ;[0, T] ; \mathbb{C})$, satisfying $R+P \star R=R+R \star P=P$.

Considering separately each of two last equalities and adding $-I$ on both sides, we varify that $(I+P)^{-1}=I-R$. Consequently, $I-(1 / \lambda) Q$ is invertible and, hence, all nonzero $\lambda \in \mathbb{C}$, are outside $\sigma(Q)$ for $Q \star \in V(C ;[0, T] ; \mathbb{C})$.

In [14, Theorem 1.2., A.2.2 ] or [15, 18.6$]$ it claims that the spectrum $\sigma(Q)$ of an element $Q \in \mathcal{A}$ is nonempty. Hence, only $\lambda=0$ belongs to the spectrum, and this implies that the spectral radius $\rho(Q)$ for kernels from $V(C ;[0, T] ; \mathbb{C})$ is zero. 
Furthermore, the following spectral radius formula

$$
\rho(Q)=\lim _{n \rightarrow \infty}\left|\left\|(Q \star)^{n}\right\|\right|^{1 / n}
$$

is known from the theorem with the same title (see [14, Theorem 2, A.2.3 ] or [15, Theorem 18.9]). This means,

$$
\lim _{n \rightarrow \infty}\left|\left\|(Q \star)^{n}\right\|\right|_{V(C ;[0, T] ; C)}^{1 / n}=0 \quad \text { for } Q \star \in V(C ;[0, T] ; \mathbb{C}) .
$$

Let us give a justification of the assumptions of Theorem 5.3 on $Q \star \in V(C ;[0, T] ; \mathbb{C})$. Since $V(C ;[0, T] ; \mathbb{C})$ is an algebra, $(Q \star)^{n} \in V(C ;[0, T] ; \mathbb{C})$, for any $n=2,3, \ldots$ According to Corollary $4.7, V(C ;[0, T] ; \mathbb{C}) \hookrightarrow \mathcal{L}(C([0, T], \mathbb{C}))$, and we can estimate

$$
\left\|(Q \star)^{n}\right\|_{\mathcal{L}(C[0, T])} \leq\|\|(Q \star)^{n} \|\left.\right|_{V(C ;[0, T] ; C)} .
$$

According to $(5.5)$, condition $(5.1)$ is satisfied for $V(C ;[0, T] ; \mathbb{C})$, completed by the unit from $\mathcal{L}(\mathcal{S}[0, T])$.

Since the assumptions of Theorem 5.3 are satisfied, for each $\lambda \in \mathbb{C} \backslash\{0\}$ and $Q \star \in$ $V(C ;[0, T] ; \mathbb{C})$,

$$
\underline{\widetilde{R}}(\lambda) \equiv(\lambda I-Q \star)^{-1}=\sum_{j=0}^{\infty} \frac{\left(Q^{\star}\right)^{j}}{\lambda^{j+1}}
$$

\section{Solvability of the Volterra Integral Equations in Banach Spaces}

We will use an idea of [1, Lemma 2.7], in which a class of Volterra operators depending on a complex parameter on a strip is considered, and which claims that their resolvents on a finite segment exist and belong to the same operator class. The idea is to reduce the proof of the Neumann series convergence for such Volterra operators to the convergence for corresponding scalar Volterra operators by taking supremum from kernels w.r.t. the complex parameter.

Lemma 6.1. Let $X$ be a Banach space, $K \star \in V(C ;[0, T] ; \mathcal{L}(X)), n, m=1,2, \ldots$ Then, for each $n \geq m$ one can estimate

$$
\left\|\sum_{j=m}^{n}(-K \star)^{j}\right\|_{\mathcal{L}(\mathcal{S}([0, T], X), C([0, T], X))} \leq \| \sum_{j=m}^{n}\left(\|K\|_{\left.\mathcal{L}(X)^{\star}\right)^{j}} \|_{\mathcal{L}(C[0, T])},\right.
$$

where $\mathcal{L}(C[0, T]) \equiv \mathcal{L}(C([0, T], \mathbb{R}))$. 
Proof. Corollary 4.7 claims that, since $K \star \in V(C ;[0, T] ; \mathcal{L}(X))$ and $F \in \mathcal{S}([0, T], X), K \star F \in$ $C([0, T], X)$. We can estimate

$$
\begin{aligned}
& \left\|\sum_{j=m}^{n}(-K \star)^{j}\right\|_{\mathcal{L}(\mathcal{S}([0, T], X), C([0, T], X))}=\sup _{\|F\|_{\mathcal{S}([0, T], X)} \leq 1}\left\|\sum_{j=m}^{n}(-1)^{j}\left[(K \star)^{j} F\right]\right\|_{C([0, T], X)} \\
& \leq \sup _{\|F\|_{\mathcal{S}([0, T], X)} \leq 1}\left\|\sum_{j=m}^{n}\right\|\left[(K \star)^{j} F\right]\|X\|_{C([0, T])} \\
& =\sup _{\|F\|_{\mathcal{S}([0, T], X)} \leq 1}\left\|\sum_{j=m}^{n}\right\|\left[K \star\left[(K \star)^{j-1} F\right]\right]\|X\|_{C([0, T])} \\
& \underset{\|F\|_{\mathcal{S}([0, T], X)} \leq 1}{\text { Corollary } 4.6} \sup _{j=m}\left\|\sum_{j=m}^{n}\right\| K\left\|_{\mathcal{L}(X)} \star\right\|\left[(K \star)^{j-1} F\right]\|X\|_{C([0, T])} \\
& \stackrel{\text { induction }}{\leq} \sup _{\|F\|_{\mathcal{S}(0, T], X)} \leq 1}\left\|\sum_{j=m}^{n}\left(\|K\|_{\mathcal{L}(X)^{\star}}\right)^{j}\right\| F\|X\|_{C([0, T])} \\
& \leq \sup _{\|F\|_{\mathcal{S}([0, T], X)} \leq 1}\left\|\sum_{j=m}^{n}\left(\|K\|_{\mathcal{L}(X)^{\star}}\right)^{j}\right\|_{\mathcal{L}(C[0, T])}\|F\|_{\mathcal{S}([0, T], X)}, \\
& =\left\|\sum_{j=m}^{n}\left(\|K\|_{\mathcal{L}(X)^{\star}}\right)^{j}\right\|_{\mathcal{L}(C[0, T]) .}
\end{aligned}
$$

Theorem 6.2. Let $X$ be a Banach space, $K \star \in V(C ;[0, T] ; \mathcal{L}(X))$ and $F \in \mathcal{S}([0, T], X)$. Then there exists a unique global solution of the problem

$$
u(t)+[K \star u](t)=F(t),
$$

in $\mathcal{S}([0, T] ; X)$, which depends continuously on $F$, that is,

$$
\|u\|_{\mathcal{S}([0, T], X)} \leq C\|F\|_{\mathcal{S}([0, T], X)},
$$

where the constant $C=C\left(\|K\|_{\mathcal{L}(X)} \star\right)$ is independent of $F$. Morover, if $\|K(t, \tau)\|_{\mathcal{L}(X)} \leq k(t, \tau) \forall t$ and a.a. $\tau \in[0, T]$, where $k \star \in V(C ;[0, T] ; \mathbb{R})$, then $C\left(\|K\|_{\mathcal{L}(X)} \star\right) \leq \tilde{C}(k \star)<\infty$.

Proof. We will look for a solution to (6.3) in the form of the Neumann series applied to the right handside function $F(t)$ :

$$
v(t):=[\underline{R} F](t) \equiv \sum_{j=0}^{\infty}(-1)^{j}\left[(K \star)^{j} F\right](t) .
$$


According to Corollary 4.7, $K \star F \in C([0, T], X)$, since $K \star \in V(C ;[0, T] ; \mathcal{L}(X))$ and $F \in$ $\mathcal{S}([0, T], X)$. Hence, all terms of series (6.5), except of the zero-term, belong to $C([0, T], X)$. As for the zero-term, $(K \star)^{0}=I$, where $I$ is an identity operator over $\mathcal{S}[0, T]$. And $I F \in$ $\mathcal{S}([0, T], X)$, since $F \in \mathcal{S}([0, T], X)$. Our aim is to show, that the Banach-valued Neumann's series $\sum_{j=1}^{\infty}(-1)^{j}(K \star)^{j}(t)$ converges w.r.t. the norm in $\mathcal{L}(\mathcal{S}([0, T], X), C([0, T], X))$. The spaces $L^{\infty}([0, T], X)$ and $C([0, T], X)$ are complete as Banach spacesm, that is, each Cauchy sequence converges. It is known, that the space of bounded linear operators mapping from a Banach space into a Banach space is also Banach space. Hence, it is enough to show that

$$
S_{n} \equiv \sum_{j=0}^{n}(-1)^{j}(K \star)^{j}
$$

is a Cauchy sequence, that is, that for any $\epsilon$ there exists a number $N$ such that $\left\|S_{n}-S_{m}\right\|_{\mathcal{L}(\mathcal{S}([0, T], X), \mathcal{C}([0, T], X))}<\epsilon$ for all $n \geq m \geq N$. We can estimate

$$
\begin{aligned}
\left\|S_{n}-S_{m}\right\|_{\mathcal{L}(\mathcal{S}([0, T], X), C([0, T], X))} \equiv & \left\|\sum_{j=m}^{n}(-1)^{j}(K \star)^{j}\right\|_{\mathcal{L}(\mathcal{S}([0, T], X), C([0, T], X))} \\
& \stackrel{\text { Lemma } 6.1}{\leq}\left\|\sum_{j=m}^{n}\left(\|K\|_{\mathcal{L}(X)} \star\right)^{j}\right\|_{\mathcal{L}(C[0, T])} .
\end{aligned}
$$

Note that, according to Corollary 4.6, $\|K\|_{\mathcal{L}(X)} \star \in V(C ;[0, T] ; \mathbb{R})$ since $K \star \in$ $V(C ;[0, T] ; \mathcal{L}(X, X))$. If the real-valued series $\underline{\tilde{R}} \equiv \sum_{j=0}^{\infty}\left(\|K\|_{\mathcal{L}(X)}\right)^{j}$ is convergent and bounded in $\mathcal{L}(C[0, T])$, there must exist a number $N$ such that $\forall n, m \geq$ $N,\left\|\sum_{j=m}^{n}\left(\|K\|_{\mathcal{L}(X)} \star\right)^{j}\right\|_{\mathcal{L}(C[0, T])}<\epsilon$. The sequence of the partial sums (6.6) will be fundamental in $\mathcal{L}(\mathcal{S}([0, T], X), C([0, T], X))$.

Let us recall Lemma 5.4 for $Q \equiv-\|K\|_{\mathcal{L}(\mathrm{X})}$ and real $\lambda=-1$. Then, owing to its statement, the real-valued series $\underline{\tilde{R}}$ converges to

$$
\underline{\tilde{R}} \equiv \sum_{j=0}^{\infty}\left(\|K\|_{\mathcal{L}(X)^{\star}}\right)^{j}=\left[I-\|K\|_{\mathcal{L}(X)^{\star}}\right]^{-1} .
$$

Owing to Lemma 5.4 , the operator $\underline{\tilde{R}}$ is bounded in $\mathcal{L}(C[0, T])$.

Owing to (6.7), this implies that the sequence of the partial sums (6.6) of the series (6.5) is fundamental in $\mathcal{L}(\mathcal{S}([0, T], X), C([0, T], X))$. Consequently, we get that $v(t) \in \mathcal{S}([0, T], X)$. Note that we excluded $m=0$ in Lemma 6.1, since, for $\mathcal{S}=L^{\infty}$, $(K \star)^{0} \notin \mathcal{L}(\mathcal{S}([0, T], X), C([0, T], X))$, but belongs to $\mathcal{L}(\mathcal{S}([0, T], X), \mathcal{S}([0, T], X))$. Applying an 
estimate similar to (6.1) for $m=0$,

$$
\begin{aligned}
\|v\|_{\mathcal{S}([0, T], X)} & \leq\left\|\sum_{j=0}^{n}(-K \star)^{j}\right\|_{\mathcal{L}(\mathcal{S}([0, T], X),(\mathcal{S}([0, T], X))}\|F\|_{\mathcal{S}([0, T], X)} \\
& \leq\|\tilde{R}\|_{\mathcal{L}(\mathcal{S}([0, T])}\|F\|_{\mathcal{S}([0, T], X)} .
\end{aligned}
$$

Furthermore, if there exists $k \star \in V(C ;[0, T] ; \mathbb{R})$ such that $\|K(t, \tau)\|_{\mathcal{L}(X)} \leq k(t, \tau) \forall t$ and a.a. $\tau \in[0, T]$, then, according to (6.8) and Lemma 5.4,

$$
\|\underline{\tilde{R}}\|_{\mathcal{L}(\mathcal{S}([0, T])} \leq\left\|\sum_{j=0}^{\infty}(k \star)^{j}\right\|_{\mathcal{L}(\mathcal{S}([0, T])}=\left\|[I-k \star]^{-1}\right\|_{\mathcal{L}(\mathcal{S}([0, T])}<\infty .
$$

That is, $\|\underline{\tilde{R}}\|_{\mathcal{L}(\mathcal{S}([0, T])} \leq \tilde{C}(k \star)<\infty$.

The fact that $v(t)$, given by convergent Neumann's series (6.5), is a solution of (6.3), can be checked by direct substitution:

$$
\begin{aligned}
v(t)+[K \star v](t) & =\sum_{j=0}^{\infty}(-1)^{j}\left[(K \star)^{j} F\right](t)+K \star \sum_{j=0}^{\infty}(-1)^{j}\left[(K \star)^{j} F\right](t) \\
& =\sum_{j=0}^{\infty}(-1)^{j}\left[(K \star)^{j} F\right](t)+\sum_{j=1}^{\infty}(-1)^{j-1}\left[(K \star)^{j} F\right](t)=F(t) .
\end{aligned}
$$

It remains only to prove the uniqueness of the solution to (6.3).

Suppose, $u$ is any solution to (6.3). We apply from the left the operator $\underline{R}$ introduced in (6.5) to both sides of (6.3):

$$
\begin{aligned}
\underline{R} u+\underline{R} K \star u & =\underline{R} F, \\
{[\underline{R} u](t)+[\underline{R} K \star u](t) } & =\sum_{j=0}^{\infty}(-1)^{j}\left[(K \star)^{j} u\right](t)+\sum_{j=0}^{\infty}(-1)^{j}(K \star)^{j}[K \star u](t) \\
& =\sum_{j=0}^{\infty}(-1)^{j}\left[(K \star)^{j} u\right](t)+\sum_{j=1}^{\infty}(-1)^{j-1}\left[(K \star)^{j} u\right](t)=u(t) .
\end{aligned}
$$

Hence,

$$
u(t)=[\underline{R} F](t),
$$

that is, any solution is represented by Banach-valued Neumann's series. Since $[\underline{R} F]$ belongs to $\mathcal{S}([0, T], X)$, solution $(6.5)$ is unique in $\mathcal{S}([0, T], X)$. Now, the theorem is proven completely. 
Lemma 6.3. Let $X, Y, Z$ be Banach spaces, $A_{0} \in C([0, T] ; \mathcal{L}(Y, Z))$, and let $A_{0}(t)$ be boundedly invertible uniformly in $[0, T]$, that is, there exists constant $c_{0}>0$ such that

$$
\left\|A_{0}(t)^{-1}\right\|_{\mathcal{L}(Z, Y)} \leq \frac{1}{c_{0}} \quad \forall t \in[0, T]
$$

Let $A \in C\left([0, T] ; L^{1}([0, T], \mathcal{L}(X, Z))\right)$. Then for $K \equiv A_{0}^{-1} A$ the following continuity property is valid:

$$
\int_{J}\|K(t+\Delta t, \tau)-K(t, \tau)\|_{\mathcal{L}(X, Y)} d \tau \longrightarrow 0 \quad \text { as } \Delta t \longrightarrow 0 \forall t, t+\Delta t \in J
$$

Proof. We use estimates (6.14), and the properties that $A_{0} \in C([0, T] ; \mathcal{L}(Y, Z))$ and $A \star$ belongs to $V(C ;[0, T] ; \mathcal{L}(X, Z))$ :

$$
\begin{aligned}
& \int_{J}\|K(t+\Delta t, \tau)-K(t, \tau)\|_{\perp(X, Y)} d \tau \\
& =\int_{J}\left\|A_{0}^{-1}(t+\Delta t) A(t+\Delta t, \tau)-A_{0}^{-1}(t) A(t, \tau)\right\|_{\mathcal{L}(X, Y)} d \tau \\
& =\int_{J}\left\|A_{0}{ }^{-1}(t+\Delta t)(A(t+\Delta t, \tau)-A(t, \tau))+\left(A_{0}^{-1}(t+\Delta t)-A_{0}^{-1}(t)\right) A(t, \tau)\right\|_{\mathcal{L}(X, Y)} d \tau \\
& \leq \int_{J}\left\|A_{0}^{-1}(t+\Delta t)\right\|_{\mathcal{L}(Z, Y)}\|(A(t+\Delta t, \tau)-A(t, \tau))\|_{\mathcal{L}(X, Z)} d \tau \\
& +\int_{J}\left\|A_{0}^{-1}(t+\Delta t)-A_{0}^{-1}(t)\right\|_{\mathcal{L}(Z, Y)}\|A(t, \tau)\|_{\mathcal{L}(X, Z)} d \tau \\
& \leq \frac{1}{c_{0}} \int_{J}\|A(t+\Delta t, \tau)-A(t, \tau)\|_{\mathcal{L}(X, Z)} d \tau \\
& +|\|A\||_{V(C ;[0, T] ; \mathcal{L}(X, Z))}\left\|A_{0}^{-1}(t)\left(A_{0}(t)-A_{0}(t+\Delta t)\right) A_{0}^{-1}(t+\Delta t)\right\|_{\mathcal{L}(Y)} \\
& \leq \frac{1}{c_{0}} \int_{J}\|A(t+\Delta t, \tau)-A(t, \tau)\|_{\mathcal{L}(X, Z)} d \tau \\
& +\frac{1}{c_{0}^{2}}|\|A\||_{V(C ;[0, T] ; \mathcal{L}(X, Z))}\left\|A_{0}(t+\Delta t)-A_{0}(t)\right\|_{\mathcal{L}(Y, Z)} \\
& \longrightarrow 0 \text { as } \Delta t \longrightarrow 0 \forall t, t+\Delta t \in J .
\end{aligned}
$$


Theorem 6.4. Let $X, Z$ be Banach spaces, $A_{0} \in C([0, T] ; \mathcal{L}(X, Z))$, and let $A_{0}(t)$ be boundedly invertible uniformly in $[0, T], A \star \in V(C ;[0, T] ; \mathcal{L}(X, Z))$, and $f \in \mathcal{S}([0, T] ; Z)$. Then there exists a unique global solution $u$ of the problem

$$
A_{0}(t) u(t)+[A \star u](t)=f(t)
$$

in $\mathcal{S}([0, T] ; X)$, which depends continuously on $f$, that is,

$$
\|u\|_{\mathcal{S}([0, T], X)} \leq C_{1}\|f\|_{\mathcal{S}([0, T], Z)^{\prime}}
$$

where the constant $C_{1}=C_{1}\left(\left\|A_{0}{ }^{-1}(t)\right\|_{\mathcal{L}(Z, X)},\left\|A_{0}^{-1}(t) A\right\|_{\left.\mathcal{L}(X)^{\star}\right)}\right.$ is independent of $f$.

Morover, if $\left\|A_{0}^{-1}(t)\right\|_{\mathcal{L}(Z, X)} \leq 1 / c_{0}$ and $\|A(t, \tau)\|_{\mathcal{L}(X, Z)} \leq a(t, \tau) \forall t$ and a.a. $\tau \in[0, T]$, where $c_{0}$ is a constant and $a \star \in V(C ;[0, T] ; \mathbb{R})$, then $C_{1} \leq\left(1 / c_{0}\right) \tilde{C}\left(1 / c_{0}\right) a \star<\infty$.

Proof. Since, $A_{0}(t)$ is invertible, we can rewrite (6.17) as the following: $u(t)+$ $A_{0}(t)^{-1} \int_{0}^{t} A(t, \tau) u(\tau) d \tau=A_{0}(t)^{-1} f(t)$. Observe that $A_{0}(t)^{-1}$ does not depend on $\tau$,

$$
u(t)+\int_{0}^{t} A_{0}(t)^{-1} A(t, \tau) u(\tau) d \tau=A_{0}^{-1}(t) f(t)
$$

We denote $K(t, \tau) \equiv A_{0}(t)^{-1} A(t, \tau)$ and $F(t) \equiv A_{0}(t)^{-1} f(t)$.

Let us show that the assumption of Theorem 6.2 are satisfied for (6.19), that is, that $K \star \in V(C ;[0, T] ; \mathcal{L}(X))$ and $F(t) \in \mathcal{S}([0, T], X)$. In order to prove that $K \star \in V(C ;[0, T] ; \mathcal{L}(X))$ we should show the justification of all the requirements of Definition 4.3.

(i) Since $A \star \in V(C ;[0, T] ; \mathcal{L}(X, Z)), A(t, \cdot) \in L^{1}([0, T], \mathcal{L}(X, Z)) \forall t \in[0, T]$ (see Definition 4.3). According to [12, Chapter 5, Corollary 2], $K(t, \cdot) \in$ $L^{1}([0, T], \mathcal{L}(X)) \forall t \in[0, T]$, since $A_{0}^{-1}(t)$ does not depend on $\tau$ and belongs to $\mathcal{L}(X, Z) \forall t \in[0, T]$. Hence, owing Theorem 4.1 , condition (i) of Definition 4.3 is satisfied.

(ii) Applying Lemma 6.3 for $A_{0} \in C([0, T] ; \mathcal{L}(X, Z))$ and $A \star \in V(C ;[0, T] ; \mathcal{L}(X, Z))$, in order to justify the continuity, completes the proof that $K \star \in V(C ;[0, T] ; \mathcal{L}(X))$. It remains to check, if $F \in \mathcal{S}([0, T], X)$. According to [16, Theorem 3.3], $F(t)$ belongs to $X$ for all or almost all $t \in[0, T]$. Continuity of $F$ w.r.t. $t$ for continuous $f(t)$, can be obtained in a similar way as in Lemma 6.3, taking into account the continuity of $A_{0}(t)$. Using Theorem 6.2 completes the proof.

\section{Appendix}

Proof of Lemma 3.4. Case (i). Each continuous Banach-valued function is strongly measurable and Bochner integrable. Hence, $a_{i j}^{h k} \in C\left([0, T]^{2}, L^{\infty}(\Omega)\right)$ satisfies requirement (i) of Definition 4.3 in case (i) of Example 2.1. Requirement (ii) of Definition 4.3 is obviously valid for the $a_{i j}^{h k}$. Hence, (2.7) presents kernels from $V\left(C ;[0, T] ; L^{\infty}(\Omega)\right)$. 
Case (ii). Let us denote the weak singular multipliers, $(t-\tau)^{-\alpha}, \tau^{-\beta}$ in case (ii) of Example 2.1, by $v(t, \tau)$, in such a way that

$$
v(t, \tau)= \begin{cases}(t-\tau)^{-\alpha}, & \text { if } \tau \leq t \\ 0, & \text { if } \tau>t\end{cases}
$$

or

$$
v(t, \tau)= \begin{cases}\tau^{-\beta,} & \text { if } \tau \leq t \\ 0, & \text { if } \tau>t\end{cases}
$$

The $v(t, \tau)$ are Lebesgue-measurable on $[0, T] \subset \mathbb{R}$ and integrable w.r.t. $\tau$ for all $t$. Hence, $v(t, \cdot) \in L^{1}[0, T] \forall t \in[0, T]$. According to [16], we can rewrite it as $v(t, \cdot) \in$ $\left.L^{1}([0, T], \mathbb{R})\right) \forall t \in[0, T]$.

Owing to the fact that $v=0$ for $\tau>t$, and considering arbitrary $t, t+\Delta t \in$ $[0, T]$, let us perform now integration over $\tau$ and show that $v \in C\left([0, T], L^{1}([0, T], \mathbb{R})\right)$. For $v$ corresponding to the $(t-\tau)^{-\alpha}$, we get $\int_{0}^{T}|v(t+\Delta t, \tau)-v(t, \tau)| d \tau=\int_{0}^{t}(t-\tau)^{-\alpha} d \tau-$ $\int_{0}^{t}(t+\Delta t-\tau)^{-\alpha} d \tau+\int_{t}^{t+\Delta t}(t+\Delta t-\tau)^{-\alpha} d \tau=1 /(1-\alpha)\left(-\left.(t-\tau)^{1-\alpha}\right|_{0} ^{t}+\left.(t+\Delta t-\tau)^{1-\alpha}\right|_{0} ^{t}-\right.$ $\left.\left.(t+\Delta t-\tau)^{1-\alpha}\right|_{t} ^{t+\Delta t}\right)=1 /(1-\alpha)\left(t^{1-\alpha}+\Delta t^{1-\alpha}-(t+\Delta t)^{1-\alpha}+\Delta t^{1-\alpha}\right) \rightarrow 0$ as $\Delta t \rightarrow 0$.

And for $v$ corresponding to the $\tau^{-\beta}$, we calculate: $\int_{0}^{T}|v(t+\Delta t, \tau)-v(t, \tau)| d \tau=$ $\int_{0}^{t+\Delta t}(\tau)^{-\beta} d \tau-\int_{0}^{t}(\tau)^{-\beta} d \tau=\int_{t}^{t+\Delta t}(\tau)^{-\beta} d \tau=1 /\left.(1-\beta)(\tau)^{1-\beta}\right|_{t} ^{t+\Delta t}=1 /(1-\beta)\left((t+\Delta t)^{1-\beta}-t^{1-\beta}\right) \rightarrow$ 0 as $\Delta t \rightarrow 0$. Owing to Definition $4.3, v \star \in V(C ;[0, T] ; \mathbb{R})$.

One can consider $v$ as a linear operator $v(t, \tau): L^{\infty}(\Omega) \rightarrow L^{\infty}(\Omega)$ defined for any function $g \in L^{\infty}(\Omega)$, on $[0, T]^{2} \backslash(\{t=\tau\} \cup\{\tau=0\})$, as following: $g \rightarrow$ $v(t, \tau) g$. Obviously, $v \in C\left([0, T], L^{1}\left([0, T], \mathcal{L}\left(L^{\infty}(\Omega), L^{\infty}(\Omega)\right)\right)\right)$, that is, $v \star \in V(C ;[0, T]$; $\left.\mathcal{L}\left(L^{\infty}(\Omega), L^{\infty}(\Omega)\right)\right)$. The functions $A_{i j, 1}^{h k}$ are in $C\left([0, T], L^{\infty}(\Omega)\right), \forall t \in[0, T]$. Owing to Corollary 4.2, $v(t, \cdot) A_{i j_{l}}^{h k}(t, \cdot) \in L^{1}\left([0, T], L^{\infty}(\Omega)\right) \forall t[0, T]$. Applying Lemma 6.3 one can varify that $v A_{i j_{l}}^{h k} \in C\left([0, T], L^{1}\left([0, T], L^{\infty}(\Omega)\right)\right.$. According to Definition $4.3, v A_{i j_{l}}^{h k} \in$ $V\left(C ;[0, T] ; \mathcal{L}\left(\mathbb{R}, L^{\infty}(\Omega)\right)\right)$, or, what is the same: $A_{i j}^{h k} \star \in V\left(C ;[0, T] ; L^{\infty}(\Omega)\right)$.

Proof of Lemma 3.3. Let us start with the proof of (3.10). The bilinear form $a_{0}(\widehat{u}, v)$ (introduced in Definition 3.1) is symmetric, owing to conditions (2.5), and continuous as follows from (2.6), that is, $\forall v \in \mathbf{H}_{0}^{1}\left(\Omega, \partial \Omega_{u}\right)$,

$$
\begin{aligned}
\left|a_{0}(\widehat{u}, v)(t)\right|: & =\left|\int_{\Omega} a_{i j 0}^{h k}(x, t) \frac{\partial \widehat{u}_{j}(x, t)}{\partial x_{k}} \frac{\partial v_{i}(x)}{\partial x_{h}} d x\right| \\
& \leq \max _{i, j, k, h}\left\|a_{i j 0}^{h k}(\cdot, t)\right\|_{L^{\infty}(\Omega)}\|\widehat{u}(t)\|_{H^{1}(\Omega)}\|v\|_{H^{1}(\Omega)} \\
& \stackrel{(2.6)}{\leq} C_{0}\|\widehat{u}(t)\|_{H^{1}(\Omega)}\|v\|_{H^{1}(\Omega)}, \quad \forall t \in[0, T] .
\end{aligned}
$$


Function $\widehat{u}:=u-\widetilde{\psi}$ must satisfy the integral identity (3.2) for any $v \in \mathbf{H}_{0}^{1}\left(\Omega, \partial \Omega_{u}\right)$. Due to symmetry (2.5), positivity (2.6) conditions, and to Korn's inequality (see [20, Chapter 1, Theorem 2.7]), the positivity condition required by the Lax-Milgram Theorem (see [16]) is satisfied:

$$
\begin{aligned}
a_{0}(\widehat{u}, \widehat{u}) & \equiv \int_{\Omega} a_{i j 0}^{h k} \frac{\partial \widehat{u}_{j}}{\partial x_{k}} \frac{\partial \widehat{u}_{i}}{\partial x_{h}} d x \\
& \stackrel{(2.5)}{=} \int_{\Omega} a_{i j 0}^{h k} \frac{1}{2}\left(\frac{\partial \widehat{u}_{j}}{\partial x_{k}}+\frac{\partial \widehat{u}_{k}}{\partial x_{j}}\right) \frac{1}{2}\left(\frac{\partial \widehat{u}_{i}}{\partial x_{h}}+\frac{\partial \widehat{u}_{h}}{\partial x_{i}}\right) d x \\
& \stackrel{(2.6)}{\geq} \frac{c_{0}}{4} \int_{\Omega}\left(\frac{\partial \widehat{u}_{j}}{\partial x_{k}}+\frac{\partial \widehat{u}_{k}}{\partial x_{j}}\right)\left(\frac{\partial \widehat{u}_{j}}{\partial x_{k}}+\frac{\partial \widehat{u}_{k}}{\partial x_{j}}\right) d x \\
& \stackrel{(\text { Korn })}{\geq} \underline{c_{0}}\|\widehat{u}\|_{H^{1} \Omega}^{2} .
\end{aligned}
$$

The assumptions of the Lax-Milgram Theorem are satisfied, and its application completes the proof for a fixed $t \in[0, T]$. Owing to the definition of the norm of $C\left([0, T], L^{\infty}(\Omega)\right)$ by $\sup _{t \in[0, T]}\|\cdot\|_{L^{\infty}(\Omega)}, A_{0 x}$ is boundedly invertible uniformly in $t$ on $[0, T]$. In order to check the continuity of $A_{0 x}$, that is, that $A_{0 x} \in C\left([0, T] ; \mathcal{L}\left(\mathbf{H}_{0}^{1}\left(\Omega, \partial \Omega_{u}\right), H^{-1}\right)\right)$, let us consider $t, t+\Delta t \in[0, T]$. Owing to (A.3),

$$
\begin{aligned}
& \left\|A_{0 x}(t+\Delta t)-A_{0 x}(t)\right\|_{\mathcal{L}\left(H_{0}^{1}(\Omega), H^{-1}\right)} \\
& \quad \leq \max _{i, j, k, h}\left\|a_{i j_{0}}^{h k}(\cdot, t+\Delta t)-a_{i j_{0}}^{h k}(\cdot, t)\right\|_{L^{\infty}(\Omega)} \longrightarrow 0 \quad \text { as } \Delta t \longrightarrow 0 .
\end{aligned}
$$

In order to prove statement (ii) of Lemma 3.3, we first have to demonstrate that the linear operator $A_{x}(t, \tau): \mathbf{H}_{0}^{1}\left(\Omega, \partial \Omega_{u}\right) \rightarrow H^{-1}(\Omega)$ is bounded for each $t$ and almost all $\tau \in$ $[0, T]$.

Let us consider the bilinear form $a(\widehat{u}, v)$ :

$$
\begin{aligned}
|a(\widehat{u}, v)(t, \tau)| & \equiv\left|\int_{\Omega} a_{i j}^{h k}(t, \tau) \frac{\partial \widehat{u}_{j}(\tau)}{\partial x_{k}} \frac{\partial v_{i}}{\partial x_{h}} d x\right| \\
& \leq \int_{\Omega}\left|a_{i j}^{h k}(t, \tau) \frac{\partial \widehat{u}_{j}(\tau)}{\partial x_{k}} \frac{\partial v_{i}}{\partial x_{h}}\right| d x \\
& \leq \max _{i, j, k, h}\left\|a_{i j}^{h k}(t, \tau)\right\|_{L^{\infty}(\Omega)}\|\widehat{u}(\tau)\|_{H^{1}(\Omega)}\|v\|_{H^{1}(\Omega)},
\end{aligned}
$$

$\forall t$ and almost all $\tau \in[0, T]$. Owing to estimate (A.6), the mapping $v \rightarrow a(\widehat{u}, v)$, for each fixed $\widehat{u}(\cdot, t) \in \mathbf{H}_{0}^{1}\left(\Omega, \partial \Omega_{u}\right)$, is a linear continuous functional on $\mathbf{H}_{0}^{1}\left(\Omega, \partial \Omega_{u}\right)$, for all $t$ and almost all $\tau \in[0, T]$. According to [21, Lemma 6.5.1], $\left\|A_{x}(t, \tau) \mathcal{L}\right\|_{\left(H_{0}^{1}\left(\Omega, \partial \Omega_{u}\right), H^{-1}\right)} \leq$ $\max _{i, j, k, h}\left\|a_{i j}^{h k}(t, \tau)\right\|_{L^{\infty}(\Omega)} \forall t$, a.a. $\tau \in[0, T]$. At the following stage, it should be shown that $A_{x}(t, \tau)$ is strongly measurable and $\left\|A_{x}(t, \tau)\right\|_{\mathcal{L}\left(H_{0}^{1}\left(\Omega, \partial \Omega_{u}\right), H^{-1}\left(\Omega, \partial \Omega_{u}\right)\right)}$ is integrable w.r.t. $\tau$ for 
all $t$ in $[0, T]$. Since $a_{i j}^{h k}(t, \cdot) \in L^{1}\left([0, T], L^{\infty}(\Omega)\right) \forall t \in[0, T]$, it can be considered as a linear operator $a_{i j}^{h k}(t, \tau): L^{2}(\Omega) \rightarrow L^{2}(\Omega) \forall t$ and a.a. $\tau \in[0, T]$. That is, we can rewrite that $a_{i j}^{h k}(t, \cdot) \in L^{1}\left([0, T], \mathcal{L}\left(L^{2}(\Omega), L^{2}(\Omega)\right) \forall t \in[0, T]\right.$. The operator $A_{x}(t, \tau)$ can be represented in the form $A_{x}(t, \tau)=\left[K_{2} f K_{1}\right](t, \tau)$, where $K_{1}:=\partial / \partial x_{k} \in \mathcal{L}\left(H_{0}^{1}\left(\Omega, \partial \Omega_{u}\right), L^{2}(\Omega)\right), f:=a_{i j}^{h k}(t, \cdot) \in$ $L^{1}\left([0, T], \mathcal{L}\left(L^{2}(\Omega), L^{2}(\Omega)\right) \quad \forall t \in[0, T]\right.$, and $K_{2} \in \mathcal{L}\left(L^{2}(\Omega), H^{-1}\left(\Omega, \partial \Omega_{u}\right)\right)$ maps $w_{i} \in L^{2}(\Omega)$ in the functionals $\int_{\Omega} w_{i}(x)\left(\partial v_{j} / \partial x_{h}\right) d x$ defined on all $v_{j} \in H_{0}^{1}\left(\Omega, \partial \Omega_{u}\right)$. Application of [12, Chapter 5, Section 5, Corollary 2] w.r.t. the operator $K_{2}$, acting on $f K_{1}$, verifies that $A_{x}(t, \cdot) \in L^{1}\left([0, T], \mathcal{L}\left(\mathbf{H}_{0}^{1}\left(\Omega, \partial \Omega_{u}\right), H^{-1}\left(\Omega, \partial \Omega_{u}\right)\right)\right), \forall t \in[0, T]$.

Let us show that $\left\|A_{x}(t, \cdot)\right\|_{L^{1}\left([0, T], \mathcal{L}\left(H^{1}(\Omega), H^{-1}\right)\right.}$ is continuous in the $t$-variable:

$$
\begin{aligned}
& \int_{0}^{t+\Delta t}\left\|A_{x}(t+\Delta t, \tau)-A_{x}(t, \tau)\right\|_{\mathcal{L}\left(H_{0}^{1}\left(\Omega, \partial \Omega_{u}\right), H^{-1}\right)} d \tau \\
& \quad \leq \int_{0}^{t+\Delta t} \max _{i, j, k, h}\left\|a_{i j}^{h k}(\cdot, t+\Delta t, \tau)-a_{i j}^{h k}(\cdot, t, \tau)\right\|_{L^{\infty}(\Omega)} d \tau \longrightarrow 0 \quad \text { as } \Delta t \longrightarrow 0
\end{aligned}
$$

for all $t+\Delta t \in[0, T]$, since $a_{i j}^{h k} \in C\left([0, T], L^{1}\left([0, T] ; L^{\infty}(\Omega)\right)\right)$.

Remark A.1. Owing to the estimate (3.11), the condition $\left\{a_{i j}^{h k}(x, t, \tau)=0\right.$ if $\left.\tau>t, \forall x \in \Omega\right\}$ additionally implies that $A_{x} \star \in V\left(C ;[0, T] ; \mathcal{L}\left(\mathbf{H}_{0}^{1}\left(\Omega, \partial \Omega_{u}\right), H^{-1}\left(\Omega, \partial \Omega_{u}\right)\right)\right)$, that is, $A_{x} \star$ is the Volterra integral operator.

Proof of Theorem 3.2. The statement of the theorem coincides with the one of Theorem 6.4 if it takes in its counterparts $\mathcal{S}:=C, X:=\mathbf{H}_{0}^{1}\left(\Omega, \partial \Omega_{u}\right)$ and $Z:=H^{-1}$.

\section{Acknowledgments}

This research has been carried out in a framework of the author's Ph.D. thesis finished in 2000. Main ideas presented in this work were particularly suggested by Professor S. E. Mikhailov from Glasgow Caledonian University. His help is highly acknowledged.

\section{References}

[1] S. E. Mikhailov, "On some weighted Hardy type classes of one-parametric holomorphic functionsII: partial volterra operators in parameter," Journal of Mathematical Analysis and Applications, vol. 222, no. 2, pp. 374-396, 1998.

[2] N. Kh. Arutyunyan and B. A. Shorkhet, "Asymptotic behavior of the solution of the boundary value problem of the theory of creep of inhomogeneous aging bodies with unilateral constraints," Mechanics of Solids, vol. 16, no. 3, pp. 31-48, 1981.

[3] P. Acquistapace, "Existence and maximal time regularity for linear parabolic integro-differential equations," Journal of Integral Equations, vol. 10, no. 1-3, pp. 5-43, 1985.

[4] A. Friedman and M. Shinbrot, "Volterra integral equations in Banach space," Transactions of the American Mathematical Society, vol. 126, pp. 131-179, 1967.

[5] A. Lunardi, "Regular solutions for time dependent abstract integro-differential equations with singular kernel," Journal of Mathematical Analysis and Applications, vol. 130, no. 1, pp. 1-21, 1988.

[6] J. Prüss, "On resolvent operators for linear integro-differential equations of Volterra type," Journal of Integral Equations, vol. 5, no. 3, pp. 211-236, 1983.

[7] G. Di Blasio, "Nonautonomous integro-differential equations in $L^{p}$ spaces," Journal of Integral Equations, vol. 10, no. 1-3, pp. 111-121, 1985. 
[8] R. C. Grimmer, "Resolvent operators for integral equations in a Banach space," Transactions of the American Mathematical Society, vol. 273, no. 1, pp. 333-349, 1982.

[9] H. Tanabe, "Linear Volterra integral equations of parabolic type," Hokkaido Mathematical Journal, vol. 12, no. 3, part 1, pp. 265-275, 1983.

[10] H. Tanabe, "Remarks on linear Volterra integral equations of parabolic type," Osaka Journal of Mathematics, vol. 22, no. 3, pp. 519-531, 1985.

[11] R. A. Adams, Sobolev Spaces, Academic Press, Boston, Mass, USA, 1990.

[12] K. Yosida, Functional Analysis, vol. 123 of Grundlehren der Mathematischen Wissenschaften, Springer, Berlin, Germany, 6th edition, 1980.

[13] E. Zeidler, Nonlinear Functional Analysis and Its Applications. II/B, Springer, New York, NY, USA, 1993.

[14] A. N. Kolmogorov and S. V. Fomīn, Reelle Funktionen und Funktionalanalysis, VEB Deutscher Verlag der Wissenschaften, Berlin, Germany, 1975.

[15] W. Rudin, Real and Complex Analysis, chapter 1, McGraw-Hill, New York, NY, USA, 3rd edition, 1987.

[16] H. W. Alt, Lineare Funktionalanalysis, Springer, Berlin, Germany, 1992.

[17] G. Gripenberg, S.-O. Londen, and O. Staffans, Volterra Integral and Functional Equations, vol. 34 of Encyclopedia of Mathematics and Its Applications, Cambridge University Press, Cambridge, UK, 1990.

[18] I. M. Gel'fand, D. A. Raikow, and G. E. Schilow, Kommutative Normierte Algebren, VEB Deutscher Verlag der Wissenschaften, Berlin, Germany, 1964.

[19] W. Rudin, Functional Analysis, McGraw-Hill Series in Higher Mathematics, McGraw-Hill, New York, NY, USA, 1973.

[20] O. A. Oleĭnik, A. S. Shamaev, and G. A. Yosifian, Mathematical Problems in Easticity and Homogenization, vol. 26 of Studies in Mathematics and Its Applications, North-Holland, Amsterdam, The Netherlands, 1992.

[21] W. Hackbusch, Theorie und Numerik elliptischer Differentialgleichungen, B. G. Teubner, Stuttgart, Germany, 1986. 


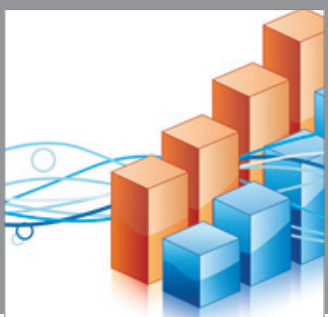

Advances in

Operations Research

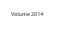

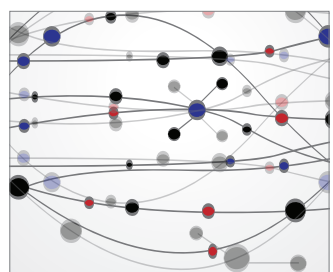

\section{The Scientific} World Journal
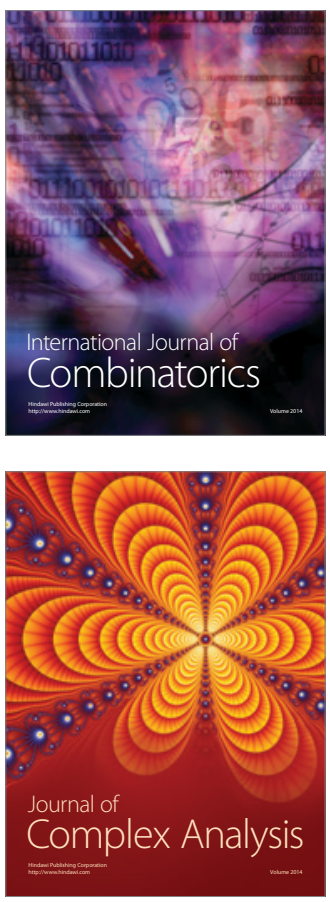

International Journal of

Mathematics and

Mathematical

Sciences
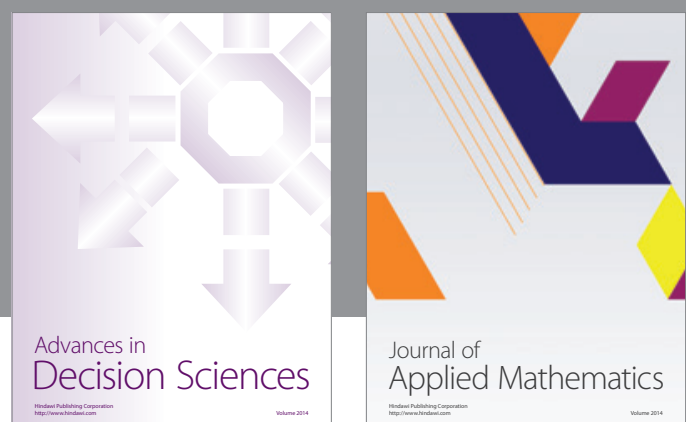

Journal of

Applied Mathematics
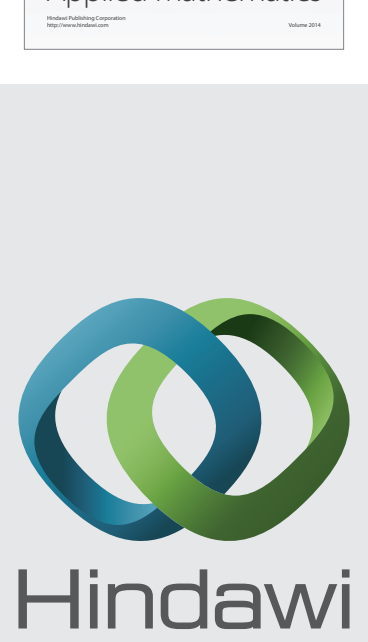

Submit your manuscripts at http://www.hindawi.com
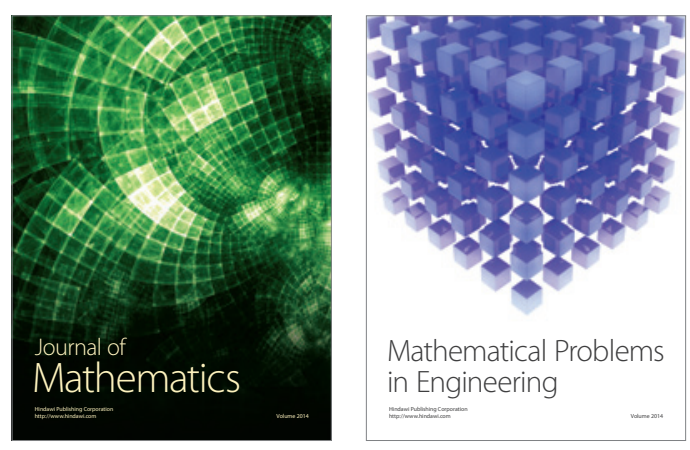

Mathematical Problems in Engineering
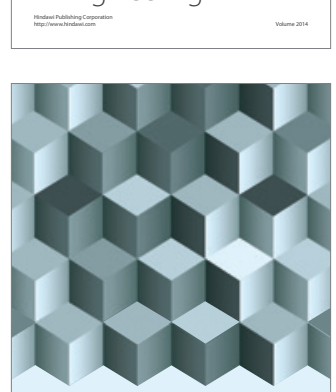

Journal of

Function Spaces
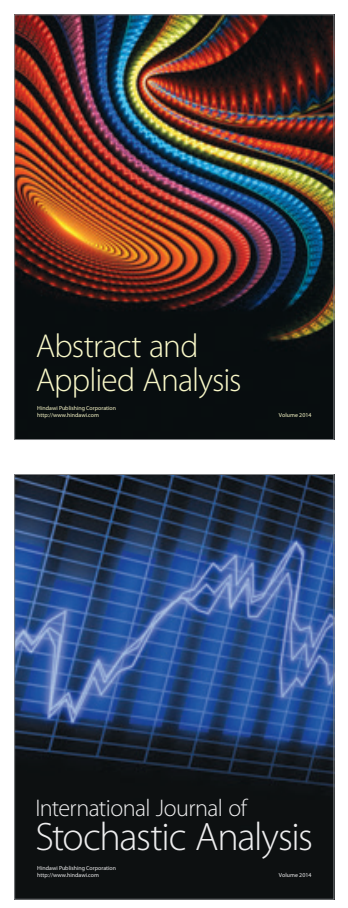

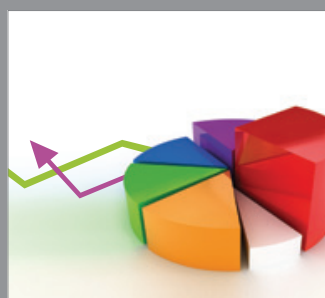

ournal of

Probability and Statistics

Promensencen
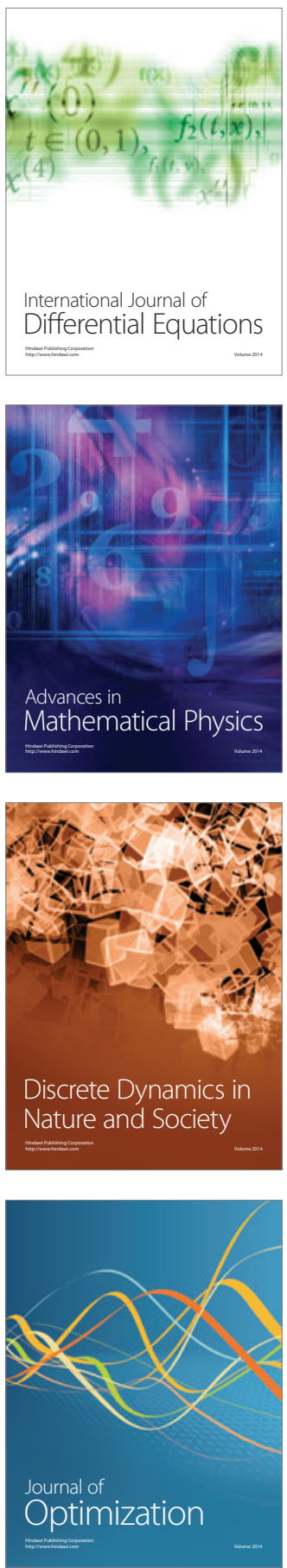